\title{
Fracture Toughness Evaluated by Indentation Methods and Its Relation to Surface Energy in Silicon Single Crystals*1
}

\author{
Masaki Tanaka $^{1, * 2}$, Kenji Higashida ${ }^{1}$, Hideharu Nakashima $^{2}$, Hidenari Takagi ${ }^{3, * 3}$ and Masami Fujiwara ${ }^{4}$ \\ ${ }^{1}$ Department of Materials Science and Engineering, Faculty of Engineering, Kyushu University, Fukuoka 812-8581, Japan \\ ${ }^{2}$ Department of Molecular and Materials Science, Graduate School of Engineering Sciences, Kyushu University, Fukuoka 816-8580, Japan \\ ${ }^{3}$ Department of Mechanical Engineering, College of Engineering, Nihon University, Fukushima 963-8642, Japan \\ ${ }^{4}$ Department of Physics, College of Engineering, Nihon University, Fukushima 963-8642, Japan
}

Fracture toughness of silicon crystals has been investigated by indentation methods, and their surface energy has been calculated using molecular dynamics (MD). When a conical indenter was forced into a (001) silicon wafer at room temperature, $\{110\}$ cracks were mainly introduced from the indent, indicating that fracture occurs most easily along the $\{110\}$ plane among the crystallographic planes of the $\langle 001\rangle$ zone. To confirm this orientation dependence, surface energies for those planes were computed using molecular dynamics. The surface energy calculated exhibits the minimum value of $1.50 \mathrm{~J} \cdot \mathrm{m}^{-2}$ at the $\{110\}$ plane, and it increases up to $2.26 \mathrm{~J} \cdot \mathrm{m}^{-2}$ at the $\{100\}$ plane. Fracture toughness was derived from these computed surface energies, and it was shown that $K_{\mathrm{IC}}$ value for the $\{110\}$ crack plane was the minimum among those for the planes of the $\langle 001\rangle$ zone. This result is in good agreement with that obtained by indentation fracture (IF) methods, although the absolute $K_{\text {IC }}$ values evaluated by the IF method were larger than those obtained by the calculation.

(Received December 2, 2002; Accepted February 26, 2003)

Keywords: crack, molecular dynamics, indentation fracture method, Vickers indentation

\section{Introduction}

Silicon crystals are the most popular material for micro electro mechanical systems (MEMS), where various mechanical performances are requested as a fundamental function of the system. ${ }^{1,2)}$ In order to develop such an use of silicon crystals, it is essential to establish the reliability on their mechanical property such as resistance to materials fracture. So far significant works have been made to understand the fracture behavior of silicon crystals. ${ }^{3)}$ However, there have been still remaining problems such as the controlling mechanism for their fracture toughness.

Since the main process of fracture in brittle solids is cutting atomic bonds to make new surfaces, the behavior of brittle fracture should highly depend on the surface energy of fracture planes. Therefore, if the surface energy depends on the orientation of its crystal plane, the value of fracture toughness should change according to the crystal orientations of the fractured planes. However, the orientation dependence of fracture toughness and its relation to the surface energy have not been fully clarified yet, although a few works have been made on this problem in silicon crystals. ${ }^{4}$ )

In the present study, orientation dependence of fracture toughness of silicon crystals at room temperature has been studied by using indentation methods. In addition, the orientation dependence of their surface energy has been also calculated using molecular dynamics. On the basis of these results, the relation between the fracture toughness and the surface energy in silicon crystals is discussed.

\footnotetext{
${ }^{* 1}$ This Paper was Presented at the Autumn Meeting of the Japan Institute of Metals, held in Suita, on 3 November 2002.

${ }^{* 2}$ Graduate Student, Kyushu University.

${ }^{* 3}$ Graduate Student, Nihon University.
}

\section{Experimental}

We employed Si single crystals cut out from a $\{001\}$ ptype wafer. Cracks were introduced by indentation methods at room temperature. In this study, two types of indenters were employed. Those indenters were forced into the $\{001\}$ plane to initiate cracks from the indent, and the cracks propagated along the planes of the $\langle 001\rangle$ zone (a series of planes parallel to the $\langle 001\rangle$ zone axis).

One of the indenters has a conical shape with the tip angle of 136 degrees (ULVAK-RIKO Inc., $\mu$ IT-1). ${ }^{8}$ The load for the conical indenter was set to be $2.94 \mathrm{~N}$. Since the conical indentation is geometrically isotropic to any direction on the wafer surface (see Fig. 1), it is expected that the cracks can propagate along the selected plane on which fracture most easily occurs among the crystallographic planes of the $\langle 001\rangle$ zone. Needless to say, silicon crystals are not isotropic elastically and plastically, so that even the conical indentation does not necessarily mean that stress fields around the indent are axial-symmetrical. However, according to our photoelastic observation using infrared light, the stress field around a conical indent is more isotropic than that around a Vickers indent, which indicates the validity of the conical indentation as a method to make a rough estimate of the most preferable fracture plane. Another type of indenter is a

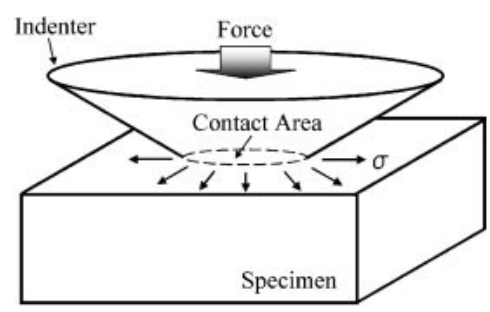

Fig. 1 Illustration of a conical indenter forced into a wafer surface. 
Vickers indenter (Akashi Co., MKV-E3), which was employed to evaluate $K_{\mathrm{IC}}$ values by the so-called indentation fracture (IF) method. ${ }^{9-15)}$ The load was set to be $0.98 \mathrm{~N}$.

Surface energies of a series of crystallographic planes parallel to the $\langle 001\rangle$ zone axis were calculated by molecular dynamics (MD). The surface energy $\gamma_{\mathrm{s}}$ is expressed by eq. (1). Here, $U_{\mathrm{b}}$ and $U_{\mathrm{s}}$ are the internal energies of a perfect crystal and a crystal with surfaces, respectively. $A$ is the surface area.

$$
\gamma_{\mathrm{s}}=\left(U_{\mathrm{b}}-U_{\mathrm{s}}\right) / 2 A
$$

The internal energies of $U_{\mathrm{b}}$ and $U_{\mathrm{s}}$ were calculated using WinMASPHYC Pro (Fujitu, Ltd.), Tersoff potential, ${ }^{16)} 0 \mathrm{~K}$ and NTP assemble. Periodic condition was applied in both calculations of $U_{\mathrm{b}}$ and $U_{\mathrm{s}}$.

TEM observations of cracks were made using a high voltage electron microscope (JEOL, JEM-1000) at the Research Laboratory of High Voltage Electron Microscopy, Kyushu University. The detail of the specimen preparation is shown in Ref. 17).

\section{Results and Discussion}

Figure 2 shows an optical microscope image around an indent introduced by a conical indenter forced into the (001) surface. The indent is observed as almost circular shape although it is chipped in the edge. Seven cracks are initiated from the indent. Among those cracks, four cracks propagating along the direction of nearly $\langle 110\rangle$ seem to be dominant. Their crack planes are almost parallel to the $\{110\}$ plane, which indicates that the $\{110\}$ plane is the most preferable fracture plane among those of the $\langle 001\rangle$ zone. In addition to these $\{110\}$ cracks, three other short cracks are also observed around the indent: they are curved and deviate from the $\{110\}$ plane. They are considered to be additional cracks which are a kind of lateral cracks usually developing in the case of the Vickers indentation. ${ }^{18)}$

Figure 3 shows a high voltage electron microscope image around the tip of a $\{110\}$ crack introduced by the indentation method at room temperature, although in this case the crack is propagating along the $\langle 100\rangle$ direction. It is to be noted here that any dislocation line segment is not found, indicating that dislocation emission can be neglected during this crack propagation. This suggests that silicon crystals observed in the present study fractured in a perfectly brittle manner. These results suggest that the $\{110\}$ plane has the minimum surface energy among the planes of the $\langle 001\rangle$ zone.

In order to verify this consideration, we made a molecular dynamics calculation for the surface energies of a series of crystallographic planes parallel to the $\langle 001\rangle$ zone axis. Figure 4 shows the orientation dependence of the surface energies calculated. The horizontal axis in the figure is the angle between the $\{110\}$ plane and the plane for which the surface energy was calculated. The perpendicular axis is the value of the surface energy. The calculation was carried out for the planes indexed $\{110\},\{320\},\{210\},\{310\},\{410\}$, $\{510\},\{610\},\{710\},\{810\}$ and $\{100\}$. It is seen from this figure that the surface energy of the $\{110\}$ plane takes the minimum value of $1.50 \mathrm{~J} \cdot \mathrm{m}^{-2}$. It simply increases from this minimum value at $\{110\}$ to the maximum value of $2.26 \mathrm{~J} \cdot \mathrm{m}^{-2}$ at $\{100\}$. The surface energy of $\{100\}$ planes is about 1.5 times larger than that of $\{110\}$ planes.

Next, we calculated the value of fracture toughness $K_{\text {IC }}$ using the surface energies obtained from the present MD calculation. When materials fracture occurs in the brittle manner, fracture toughness is calculated by eq. (2) based on the Griffith criterion: ${ }^{19)}$

$$
K_{\mathrm{IC}}=\sqrt{2 E \gamma_{\mathrm{s}} /\left(1-v^{2}\right)}
$$

where $\gamma_{\mathrm{s}}$ is surface energy, $v$ is Poisson's ratio and $E$ is Young's modulus. Here, in order to take into account the orientation dependence of the Young's modulus, we substitute $E_{[h k l]}$ given by eq. (3) for $E$ in eq. (2). ${ }^{20)}$

$$
\begin{aligned}
1 / E_{[h k l]} & =S_{11}-2\left(S_{11}-S_{12}-1 /\left(2 S_{44}\right)\right) \lambda_{\alpha \beta \theta}, \\
\lambda_{\alpha \beta \theta} & =\cos ^{2} \alpha \cos ^{2} \beta+\cos ^{2} \beta \cos ^{2} \theta+\cos ^{2} \theta \cos ^{2} \alpha, \\
\cos \alpha & =h /\left(h^{2}+k^{2}+l^{2}\right)^{1 / 2}, \cos \beta=k /\left(h^{2}+k^{2}+l^{2}\right)^{1 / 2}, \\
\cos \theta & =l /\left(h^{2}+k^{2}+l^{2}\right)^{1 / 2},
\end{aligned}
$$

where $E_{[h k l]}$ is a Young's modulus in the direction of $[h k l] ; \alpha$, $\beta$ and $\theta$ are the angles between the direction $[h k l]$ and the $\langle 100\rangle$ axes of cubic crystals; the compliances for silicon $S_{11}$, $S_{12}$ and $S_{44}$ are $0.767 \times 10^{-11},-0.213 \times 10^{-11}$ and $1.256 \times 10^{-11} \mathrm{~Pa}^{-1}$ respectively; the poison's ratio $v$ and the anisotropy factor $2 C_{44} /\left(C_{11}-C_{12}\right)$ are 0.218 and 1.56 , respectively. $^{21)}$

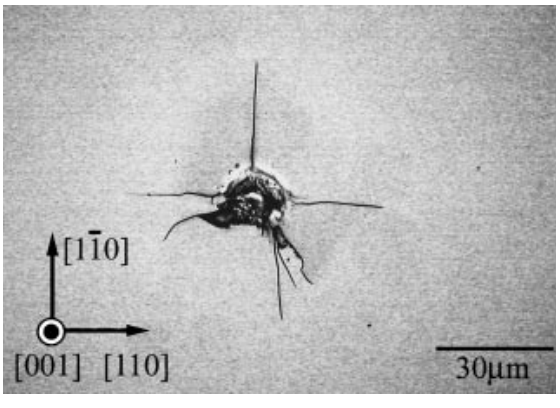

Fig. 2 Optical micrograph of an indent introduced at room temperature, using a conical indenter forced into the (001) plane. Several cracks are initiated from the indent.
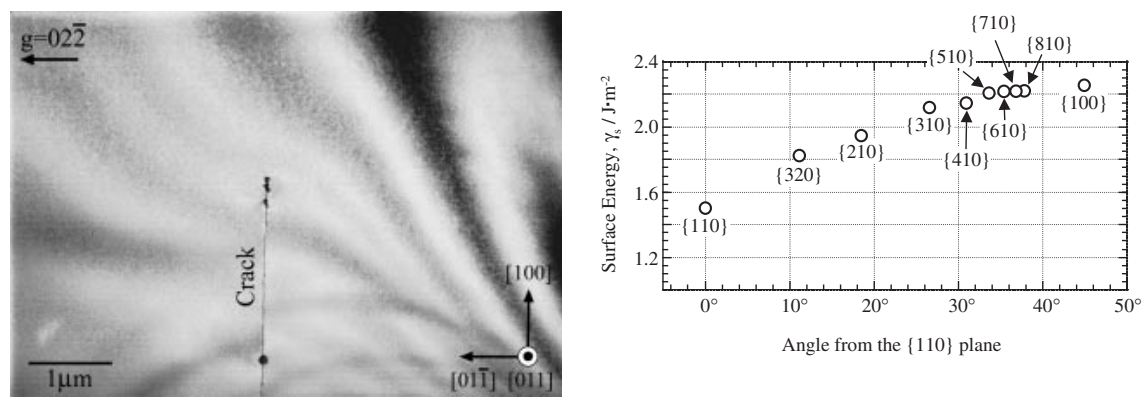

Fig. 3 HVEM image of a crack introduced by indentation method at room temperature.
Fig. 4 Orientation dependence of surface energy for crystallographic planes of the $\langle 001\rangle$ zone calculated by molecular dynamics. 


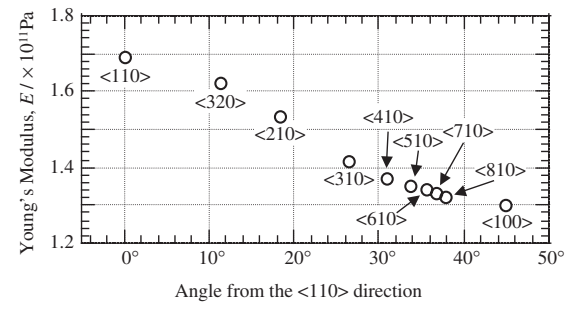

Fig. 5 Orientation dependence of Young's modulus for the directions perpendicular to $\langle 001\rangle$.

Figure 5 shows the orientation dependence of the Young's modulus for a series of directions perpendicular to the $\langle 001\rangle$ axis. Here the horizontal and vertical axes are the angle from the $\langle 110\rangle$ direction and the value of $E_{[h k l]}$, respectively. The orientation dependence of the Poisson's ratio was neglected since the contribution of $v$ to $K_{\mathrm{IC}}$ is no more than $2 \%$ comparing to that of $E_{[h k l]}$ It is to be noted here that the orientation dependence of Young's modulus exhibits an opposite tendency to that of surface energies: Young's modulus increase with the angle from $\{110\}$, while surface energies in Fig. 4 decrease in spite that it is generally thought that surface energy is an increasing function of Young's modulus. ${ }^{22)}$ Although a detailed discussion may be necessary to understand this problem, it must closely relate with the potential function of inter-atomic bonding: Young's modulus is one of the key parameters for determining surface energies, but surface energies depend not only on $E$ but also on other parameters such as interaction distance of inter-atomic bonding. The decrease of such parameter should reduce the energy to form new surfaces. Gilman showed the same tendency of surface energy of $\mathrm{Si}$ as the present result, although his result is also derived from an intuitive discussion. $^{23)}$

Figure 6 exhibits the orientation dependence of $K_{\text {IC }}$ values calculated from the surface energies and Young's modulus shown in Figs. 4 and 5. The horizontal axis in the figure is the same as that in Fig. 4, and vertical axis indicates the $K_{\text {IC }}$ value. In this figure, the fracture toughness takes the minimum value of $0.73 \mathrm{MPa} \cdot \mathrm{m}^{1 / 2}$ which was calculated under the condition that the fracture occurs along the $\{110\}$ plane. However, the $K_{\mathrm{IC}}$ values obtained from the other planes exhibit almost the same value of around $0.79 \mathrm{MPa} \cdot \mathrm{m}^{1 / 2}$, and they do not show the clear dependence on the orientation of fractured planes. This tendency is understood by taking into account the orientation dependence of Young's modulus shown in Fig. 5, where $E_{[h k l]}$ decreases

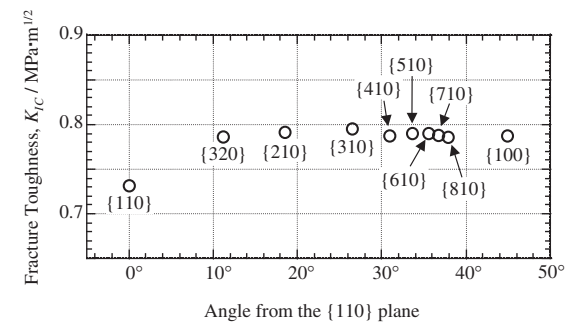

Fig. 6 Orientation dependence of $K_{\mathrm{IC}}$ values derived from the surface energies calculated for the planes of the $\langle 001\rangle$ zone. from the value for the $\langle 110\rangle$ direction to that for $\langle 100\rangle$. As understood from eq. (2), the increase of the surface energy from the value of the $\{110\}$ plane to that of $\{100\}$ is canceled by the decrease of the Young's modulus except the fracture along the $\{110\}$ plane. When the fracture plane is selected to be $\{110\}$, the decrease of the surface energy is significant comparing the increase of the elastic constant, so that the $K_{\mathrm{IC}}$ value takes the minimum value among the planes of the $\langle 001\rangle$ zone. These results are in good agreement with the fact that the $\{110\}$ cracks are dominant ones, as shown in Fig. 2.

In order to confirm quantitatively such orientation dependence of fracture toughness, we measured the $K_{\mathrm{IC}}$ values for the $\{110\}$ and the other fracture planes of the $\langle 001\rangle$ zone by using the indentation fracture method. ${ }^{9-15)}$ According to Niihara, ${ }^{13)}$ the value of fracture toughness, $K_{\mathrm{IC}}$, is expressed by eq. (4). This equation is applicable to the case of $c / a>2.5$, where $c$ and a are the size of the median crack introduced by the indent and a half of the diagonal of the Vickers indent, respectively.

$$
K_{\mathrm{IC}}=A\left(H / E_{[h k l]}\right)^{2 / 5}\left(P / c^{3 / 2}\right), \quad P \fallingdotseq H / a^{2} .
$$

where $A$ is a constant $\left(A=0.129 \phi^{-3 / 5}, \phi \fallingdotseq 3\right), H$ is the hardness, and $E_{[h k l]}$ is a Young's modulus in the direction of $[h k l]$.

In the present measurement, as shown in Fig. 7, Vickers indenter was forced into a (001) wafer surface in order to introduce the $\{110\}$ or $\{100\}$ cracks. When the diagonal direction of the indent was taken to be parallel to the $\langle 110\rangle$ direction (Fig. 7(a)), the $\{110\}$ sharp cracks were introduced from the corner of the indent. On the other hand, when the diagonal direction was taken to be $\langle 100\rangle$ (Fig. 7(b)), cracks were also introduced from the corner of the indent, but they did not propagate straightly to the expected direction of $\langle 100\rangle$. They are curved and deviate from the $\{100\}$ plane. These results can be understood by the orientation depen-

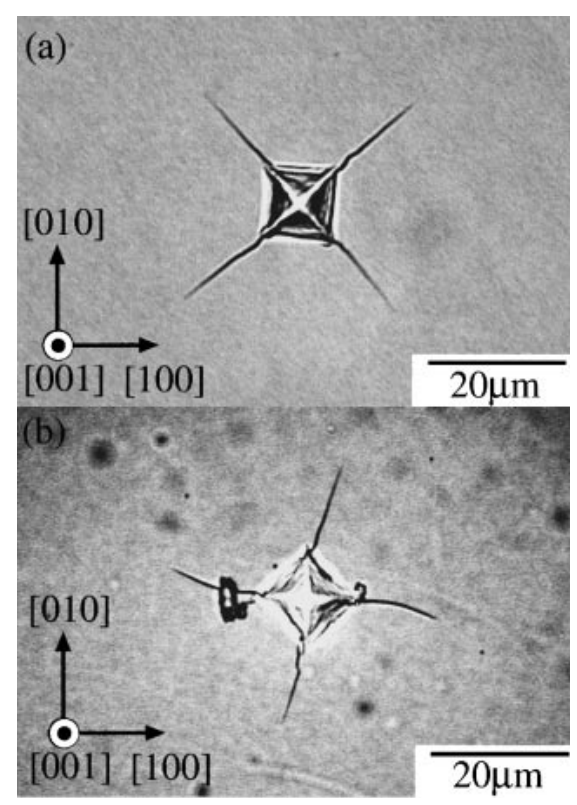

Fig. 7 Optical micrographs of indents introduced by Vickers indentation: (a) the diagonal of the indent is parallel to $\langle 110\rangle$, (b) the diagonal of the indent is parallel to $\langle 100\rangle$. 
Table 1 Data obtained by indentation fracture method.

\begin{tabular}{ccccc}
\hline Crack Plane & $\begin{array}{c}\text { Number of } \\
\text { Samples }\end{array}$ & $\begin{array}{c}\text { Average of } K_{\mathrm{IC}} \\
\left(\mathrm{MPa} \cdot \mathrm{m}^{1 / 2}\right)\end{array}$ & $\begin{array}{c}\text { Variance of } K_{\mathrm{IC}} \\
\left(\mathrm{MPa}^{2} \cdot \mathrm{m}\right)\end{array}$ & $\begin{array}{c}\text { Average of } H \\
(\mathrm{GPa})\end{array}$ \\
\hline $\begin{array}{c}\{110\} \\
\text { Planes of }\end{array}$ & 14 & 1.12 & 0.00562 & 0.7 \\
$\langle 001\rangle$ zone & 12 & 1.29 & 0.00866 & 10.6 \\
\hline
\end{tabular}

dence of the calculated $K_{\mathrm{IC}}$ values indicated in Fig. 5: the fracture toughness of the $\{100\}$ planes is almost the same as those of the other planes near $\{100\}$, while the fracture toughness of the $\{110\}$ plane takes the minimum value. It is thus understood that the $\{110\}$ crack is the sharpest among a series of cracks of the $\langle 001\rangle$ zone.

Table 1 shows the values of fracture toughness obtained using eq. (4) in the present study. In the case of Fig. 7(b), the crack planes deviate from the $\{100\}$ plane, although they are considered to be the planes of the $\langle 001\rangle$ zone. In the present paper, we call these cracks "cracks of the $\langle 001\rangle$ zone" for convenience. Fourteen IF tests were made to measure the fracture toughness for the $\{110\}$ crack, we obtained the value of $1.12 \mathrm{MPa} \cdot \mathrm{m}^{1 / 2}$ as the average value (sample variance: $\left.0.00562 \mathrm{MPa}^{2} \cdot \mathrm{m}\right)$. To measure the fracture toughness for the crack of the $\langle 001\rangle$ zone, twelve IF tests were performed. The average $K_{\mathrm{IC}}$ value for the cracks was $1.29 \mathrm{MPa} \cdot \mathrm{m}^{1 / 2}$ (sample variance: $0.00866 \mathrm{MPa}^{2} \cdot \mathrm{m}$ ). It is thus confirmed also by the IF method that the fracture toughness for the $\{110\}$ crack is less than those of the other cracks of the $\langle 001\rangle$ zone.

Remaining problem is that the absolute values of fracture toughness measured by the IF method is larger than those derived from the surface energies calculated using MD. In eq. (4), the constant $A$ was empirically determined so as to make the $K_{\text {IC }}$ values of the IF method consistent with those obtained by other $K_{\text {IC }}$ tests. Therefore, fracture toughness obtained from the IF method include the contribution of plastic deformation to $K_{\mathrm{IC}}$ values. On the other hand, fracture toughness derived from surface energies calculated by MD does not include such contribution of plastic work. It is thus considered that the $K_{\mathrm{IC}}$ values obtained by IF methods tend to show higher values than those derived from the MD calculation.

\section{Conclusion}

Orientation dependence of fracture toughness in silicon crystals at room temperature was investigated by using both an indentation method and the surface energies computed by MD. It was found by indentation methods using a conical indenter that fracture occurred most easily along the $\{110\}$ plane among the planes of the $\langle 001\rangle$ zone. Surface energies for the planes of the $\langle 001\rangle$ zone were calculated using MD, from which the $K_{\mathrm{IC}}$ values were evaluated. It was shown also by this evaluation that the $K_{\mathrm{IC}}$ values for the $\{110\}$ crack was the minimum among those for the $\langle 001\rangle$ zone. Indentation fracture tests using a Vickers indenter was also made. It was confirmed also by this method that the $K_{\mathrm{IC}}$ values for the $\{110\}$ crack was less than those for the other crack planes of the $\langle 001\rangle$ zone, although the absolute values obtained by this IF method was larger than those from the MD calculation.

It is thus concluded that the $K_{\mathrm{IC}}$ values of silicon crystals are dependent on the orientation of fracture planes, which can be understood mainly from the orientation dependence of their surface energy.

\section{REFERENCES}

1) W. Lang: Mater. Sci. Eng. R17 (1996) 1-55.

2) M. Esashi: Vacuum 47 (1996) 469-474.

3) A. E. Carlsson and R. Thomson: Solid State Physics, Ed. by H. Ehrenreich and F. Spaepen, Vol. 51 (Academic Press, 1997) pp. 233280.

4) C. P. Chen and M. H. Leipold: Am. Ceram. Soc. Bull. 59 (1980) 469472.

5) K. Hayashi, S. Tsujimoto, Y. Okamoto and T. Nishikawa: J. Soc. Mater. Sci. Japan 40 (1991) 405-410.

6) M. Kishi and Y. Miura: Int. Sym. Designing Processing and Properties of Adv. Eng. Mater., (JSPS AEM 156 committee, 1997) pp. 397-402.

7) F. Ebrahimi and L. Kalwani: Mater. Sci. Eng. A268 (1999) 116-126.

8) M. Fujiwara: J. Japan Inst. Light Met. 52 (2002) 282-290.

9) B. R. Lawn and T. R. Wilshaw: J. Mater. Sci. 10 (1975) 1049-1081.

10) B. R. Lawn, A. G. Evans and D. B. Marshall: J. Am. Ceram. Soc. 63 (1980) 574-581.

11) G. R. Anstis, P. Chantikul, B. R. Lawn and D. B. Marshall: J. Am. Ceram. Soc. 64 (1981) 533-538.

12) S. S. Chiang, D. B. Marshall and A. G. Evans: J. Appl. Phy. 53 (1982) 298-311.

13) K. Niihara: J. Mater. Sci. Let. 2 (1983) 221-223.

14) K. Tanaka, Y. Kitahara, Y. Ichinose and T. Iimura: Acta. Metall. 32 (1984) 1719-1729.

15) K. Tanaka: J. Mater. Sci. 22 (1987) 1501-1508.

16) J. Tersoff: Phys. Rev. B 39(8) (1989) 5566-5568.

17) K. Higashida, N. Narita, M. Tanaka, T. Morikawa, Y. Miura and R. Onodera: Philos. Mag. A 82 (2002) 3263-3273.

18) R. F. Cook and G. M. Pharr: J. Am. Ceram. Soc. 73 (1990) 787-817.

19) A. A. Griffith: Philos. Trans. R. Soc. London Ser. A 221 (1920) 163198.

20) A. Kelly and N. H. Macmillan: Strong Solids, 3rd edn., (Oxford Science, Oxford, 1986) pp. 382-403.

21) J. P. Hirth and J. Lothe: Theory of Dislocations 2nd Ed., (Krieger Publishing Company Malabar, Florida, 1992) pp. 836-837.

22) R. W. Davidge: Mechanical behaviour of ceramics, (Cambridge University Press, Cambridge, 1979) p. 32.

23) J. J. Gliman: Fracture, Ed. by B. L. Averbach, D. K. Felbeck, G. T. Hahn and D. A. Thomas, (The Technology Press of Massachusetts Institute of Technology, 1959) pp. 204-205. 